OPEN ACCESS

Edited by:

lan Pearce,

Manchester University NHS

Foundation Trust (MFT),

United Kingdom

Reviewed by:

Luca Mazzarella,

European Institute of Oncology (IEO),

Xiangming Cheng

Peking Union Medical College Hospital

(CAMS), China

*Correspondence: Lijin Zhang

stzlj913729553@163.com

Specialty section: This article was submitted to Surgical Oncology,

a section of the journal

Frontiers in Oncology

Received: 01 March 2020 Accepted: 22 December 2020 Published: 08 February 2021

Citation:

Zhang L, Zhao H, Wu B, Zha Z, Yuan J and Feng $Y$ (2021) Predictive Factors for Positive Surgical Margins in Patients With Prostate Cancer After Radical Prostatectomy: A Systematic Review and Meta-Analysis. Front. Oncol. 10:539592. doi: 10.3389/fonc.2020.539592

\section{Predictive Factors for Positive Surgical Margins in Patients With Prostate Cancer After Radical Prostatectomy: A Systematic Review and Meta-Analysis}

\author{
Lijin Zhang ${ }^{*}$, Hu Zhao, Bin Wu, Zhenlei Zha, Jun Yuan and Yejun Feng \\ Department of Urology, Affiliated Jiang-yin Hospital of the Southeast University Medical College, Jiang-yin, China
}

Background and Objectives: Previous studies have demonstrated that positive surgical margins (PSMs) were independent predictive factors for biochemical and oncologic outcomes in patients with prostate cancer ( $\mathrm{PCa}$ ). This study aimed to conduct a metaanalysis to identify the predictive factors for PSMs after radical prostatectomy (RP).

Methods: We selected eligible studies via the electronic databases, such as PubMed, Web of Science, and EMBASE, from inception to December 2020. The risk factors for PSMs following RP were identified. The pooled estimates of standardized mean differences (SMDs)/odds ratios (ORs) and 95\% confidence intervals (Cls) were calculated. A fixed effect or random effect was used to pool the estimates. Subgroup analyses were performed to explore the reasons for heterogeneity.

Results: Twenty-seven studies including 50,014 patients with PCa were eligible for further analysis. The results showed that PSMs were significantly associated with preoperative prostate-specific antigen (PSA) (pooled SMD $=0.37 ; 95 \% \mathrm{Cl}: 0.31-0.43$; $\mathrm{P}<0.001$ ), biopsy Gleason Score $(<6 / \geq 7)$ (pooled $\mathrm{OR}=1.53$; 95\% Cl:1.31-1.79; $\mathrm{P}<$ 0.001 ), pathological Gleason Score $(<6 / \geq 7)$ (pooled OR $=2.49$; 95\% Cl: 2.19-2.83; $\mathrm{P}<$ 0.001), pathological stage $(<\mathrm{T} 2 / \geq \mathrm{T} 3)$ (pooled $\mathrm{OR}=3.90 ; 95 \% \mathrm{Cl}: 3.18-4.79 ; \mathrm{P}<0.001)$, positive lymph node (PLN) (pooled $\mathrm{OR}=3.12 ; 95 \% \mathrm{Cl}$ : 2.28-4.27; $\mathrm{P}<0.001$ ), extraprostatic extension (EPE) (pooled $\mathrm{OR}=4.44 ; 95 \% \mathrm{Cl}$ : 3.25-6.09; $\mathrm{P}<0.001$ ), and seminal vesicle invasion (SVI) (pooled $\mathrm{OR}=4.19 ; 95 \% \mathrm{Cl}$ : 2,87-6.13; $\mathrm{P}<0.001$ ). However, we found that age (pooled SMD $=0.01 ; 95 \% \mathrm{Cl}:-0.07-0.10 ; \mathrm{P}=0.735$ ), body mass index $(\mathrm{BMI})$ (pooled $\mathrm{SMD}=0.12 ; 95 \% \mathrm{Cl}:-0.05-0.30 ; \mathrm{P}=0.162$ ), prostate volume (pooled SMD = -0.28; 95\% Cl: $-0.62-0.05 ; \mathrm{P}=0.097$ ), and nerve sparing (pooled $\mathrm{OR}=0.90 ; 95 \% \mathrm{Cl}: 0.71-1.14 ; \mathrm{P}=0.388$ ) had no effect on PSMs after RP. Besides, the findings in this study were found to be reliable by our sensitivity and subgroup analyses.

Conclusions: Preoperative PSA, biopsy Gleason Score, pathological Gleason Score, pathological stage, positive lymph node, extraprostatic extension, and seminal vesicle 
invasion are independent predictors of PSMs after RP. These results may helpful for risk stratification and individualized therapy in PCa patients.

Keywords: prostate cancer, radical prostatectomy, positive surgical margins, risk factors, meta-analysis

\section{INTRODUCTION}

Prostate cancer $(\mathrm{PCa})$ is the most common type of newly diagnosed malignancy and a leading cause of cancer-related death in males worldwide (1). With the wide use of the prostate-specific antigen (PSA) screening test, the majority of PCa patients are diagnosed in the early stages (2). As a result, radical prostatectomy (RP) with bilateral pelvic lymph node dissection has been the gold standard for the treatment of patients with localized PCa (3). The goal of RP for PCa is complete prostate extirpation; despite favorable cancer control associated with RP, approximately $25 \%$ of all patients experience biochemical recurrence (BCR) (4). A number of factors have been found to be associated with BCR after RP, and one adverse risk factor is the presence of positive surgical margins (PSMs).

PSMs are defined as an extension of cancer cells to the inked cut surface of the RP specimen (5). Our previous findings have indicated that PSMs are significantly associated with BCR and poor survival outcome after RP $(6,7)$. However, none of the systematic research studies have reported about the factors that may affect the margin status of PCa after RP. Conventional parameters for risk estimation of PSMs are mainly based on factors, including preoperative PSA ( $\mathrm{p}$ PSA), pathological T stage, pathological Gleason Score (GS), and multiple positive biopsy cores (8-11). However, the prognostic value of these predictive factors is limited. Besides, PSMs may be affected by remnant normal tissue and inadequate surgical skill (12). Therefore, no consensus has been reported regarding the above results. Based on these considerations, a comprehensive metaanalysis and systematic review was necessary to evaluate the predictive factors for PSMs in PCa patients following RP.

\section{MATERIALS AND METHODS}

\section{Literature and Search Strategy}

We carried out this meta-analysis in accordance with the guidelines of the Preferred Reporting Items for Systematic Review and Meta-Analyses statement (PRISMA) (13). A comprehensive literature search was conducted using the PubMed, Web of Science, Wanfang, and China National Knowledge Infrastructure (CNKI) databases. Search strategies were based on the combination of Medical Subject Headings (MeSH) and keywords as follows: "prostate cancer," "radical

\footnotetext{
Abbreviations: PCa, renal cell cancer; PSMs, positive surgical margins; NSMs, negative surgical margins; RP, radical prostatectomy; PRISMA, Preferred Reporting Items for Systematic Reviews and Meta-Analyses; NOS, Newcastle Ottawa scale; ORs, odds ratios; SMD, standard mean differences; CIs, corresponding confidence intervals; p-PSA, preoperative PSA; GS, Gleason Score; PLN, positive lymph node; EPE, extraprostatic extension; SVI, seminal vesicle invasion; BMI, body mass index; RE, random-effects; FE, fixed-effects.
}

prostatectomy," "positive surgical margin," "clinicopathological" and "risk factors." The last search was conducted on December 2020. Meanwhile, to identify other eligible publications, reference lists were also screened manually. The language was restricted to English and Chinese. Because we did not perform clinical research in this study, no ethical approval was needed and all analyses were based on previously published literatures.

\section{Selection Criteria and Data Extraction}

Papers were included in this meta-analysis if they met the following criteria: (1) all patients with a diagnosis of PCa and PSMs were histopathologically confirmed; (2) treatment was limited to RP; (3) clinicopathological features were analyzed according to the surgical margins status, and all studies had a comparable study design; (4) standardized mean differences (SMDs)/odds ratios (ORs) and 95\% confidence intervals (CIs) were reported in the paper or could be computed from the given data; (5) if more than one article was identified in the same cohort, the most comprehensive and largest dataset was adopted. Accordingly, studies with the following criteria were excluded: (1) case reports, review articles, editorials, and non-original articles; (2) papers not published in English and Chinese; (3) studies that did not analyze the PSMs and clinical features; (4) studies lacking sufficient data to acquire SMDs/ORs and $95 \%$ CIs. Literature search was independently performed by two investigators. Disagreement was resolved by discussion.

\section{Data Extraction and Quality Assessment}

Two researchers (BW and $\mathrm{ZZ}$ ) assessed the titles and abstracts of the searched studies, respectively. Any disagreements were reconciled by a third researcher (JY). The following information was extracted from the included studies: publication information (first author's last name, publication year, country of origin, and study design), patients' characteristics (mean age, p-PSA, and follow-up time), and PCa outcomes (tumor stage, GS, and oncologic outcomes). According to the Newcastle-Ottawa quality assessment scale (NOS) (14), two researchers (HZ and YF) independently assessed the quality of each study. According to its criteria, the NOS estimates studies based on the following three parts: selection, comparability, and outcome assessment. For quality assessment, scores ranged from 0 to 9 , and studies with scores of 6 or more were rated as being of high quality.

\section{Statistical Analysis}

For this meta-analysis, pooled SMDs/ORs with 95\% CIs were used to describe the relationship between risk factors and PSMs. An OR $>1$ or SMD $>0$ suggested a close relationship of PSMs in patients with PCa. Heterogeneity among studies was evaluated by using Cochran's Q test and Higgins $\boldsymbol{I}$-squared statistic. If the $\boldsymbol{I}^{2}$ value was $>50 \%$ or the $P_{\text {heterogeneity }}$ was $<0.1$, it suggested a statistically significant heterogeneity in the included studies, and a randomeffects (RE) model was adopted; otherwise, a fixed-effects (FE) model 
was used. To consider the potential reason for heterogeneity, subgroup analysis was conducted. To test the stability of the result, we performed a sensitivity analysis by excluding one study in turn. Visual inspection of asymmetry in funnel plots was carried out to assess the potential publication bias. Furthermore, we performed Egger's tests to provide quantitative evidence of publication bias. These statistical analyses or data syntheses were calculated using STATA version 12.0 (Stata Corporation, College Station, TX, USA). All statistical tests were two sided, and $\mathrm{P}<0.05$ was considered statistically significant.

\section{RESULTS}

\section{Literature Search}

A flowchart of the literature selection process is shown in Figure 1. The initial search of electronic databases identified 1,568 records according to the search criteria; after the duplicates were removed, 883 papers remained behind. A total of 588 papers were then excluded by screening the titles and abstracts. Then, 295 full-text articles were further examined and 268 articles were excluded because 27 articles included the same cohort of patients and 241 articles lacked enough data for further research. Finally, 27 articles $(8,15-40)$ published between 2009 and 2020 were included in this meta-analysis.

\section{Features of the Included Studies}

Summary of the major characteristics of these studies is shown in Table 1 and Table 2 . All studies had a retrospective study design. The sample size ranged from 144 to 12,515 , and a total of 50,014 patients were included. A total of 12,093 PCa patients with PSMs were included in our study, which accounted for $24.2 \%$ of all patients. Geographically, eight studies were conducted in Asia, eight in North America, eight in Europe, two in Australia, and one in multi-center locations. All patients had received RP as primary treatment for PCa. According to the NOS quality assessment, all studies included in this study were categorized as being of high quality (Supplementary Table S1).

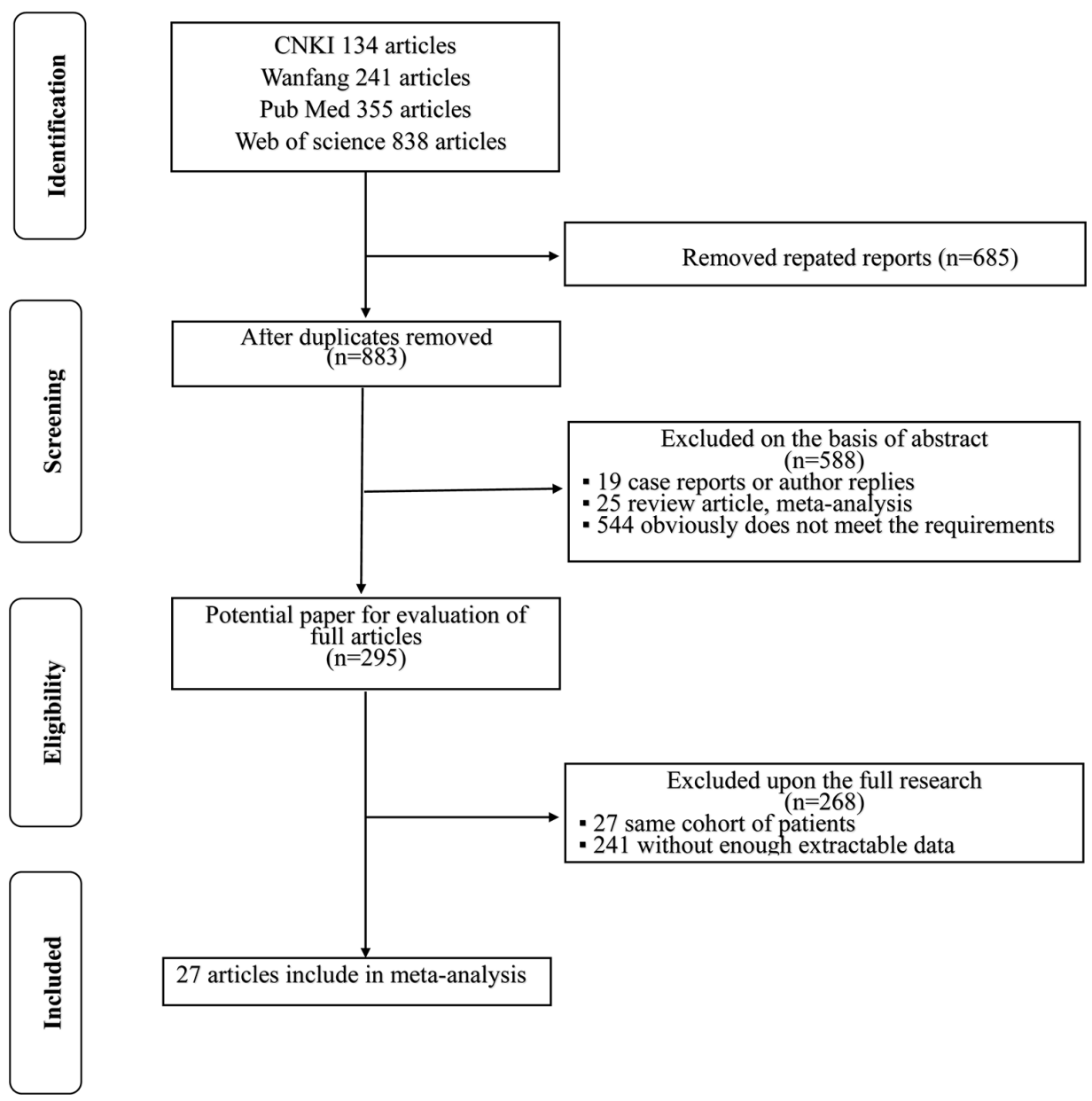

FIGURE 1 | Flowchart of the literature review process for the selection of eligible literatures. 
TABLE 1 | The basic characteristics of all studies included in this meta-analysis.

\begin{tabular}{|c|c|c|c|c|c|c|c|c|c|c|c|}
\hline \multirow[t]{2}{*}{ Author } & \multirow[t]{2}{*}{ Year } & \multirow[t]{2}{*}{ Country } & \multirow{2}{*}{$\begin{array}{l}\text { Recruitment } \\
\text { period }\end{array}$} & \multicolumn{2}{|c|}{ No. of patients } & \multicolumn{2}{|c|}{ Age (years) } & \multicolumn{2}{|c|}{ Pre-PSA } & \multicolumn{2}{|c|}{ Follow-up (months) } \\
\hline & & & & PSMs & NSMs & PSMs & NSMs & PSMs & NSMs & PSMs & NSMs \\
\hline Celik et al. (15) & 2020 & Turkey & 2005-2020 & 893 & 1,750 & $\begin{array}{c}\text { Mean } \pm \text { SD } \\
63.2 \pm 6.5\end{array}$ & $\begin{array}{c}\text { Mean } \pm \text { SD } \\
62.4 \pm 6.7\end{array}$ & $\begin{array}{c}\text { Mean } \pm \text { SD } \\
13 \pm 18.9\end{array}$ & $\begin{array}{c}\text { Mean } \pm \text { SD } \\
8.8 \pm 9.5\end{array}$ & NA & NA \\
\hline Porcaro et al. (16) & 2020 & Italy & 2013-2017 & 192 & 540 & $\begin{array}{c}\text { Median } \\
(\text { IQR) } \\
65 \text { (60-69) }\end{array}$ & $\begin{array}{c}\text { Median } \\
(\text { IQR) } \\
65(60-69)\end{array}$ & $\begin{array}{c}\text { Median } \\
\quad(\text { IQR }) \\
6.9(5.1-8.7)\end{array}$ & $\begin{array}{c}\text { Median } \\
\text { (IQR) } \\
6.1(4.8-8.3)\end{array}$ & $\begin{array}{l}\text { Median (IQR) } \\
26(14-40)\end{array}$ & $\begin{array}{c}\text { Median (IQR) } \\
26(14-40)\end{array}$ \\
\hline Tian et al. (17) & 2019 & China & 2010-2016 & 142 & 267 & $\begin{array}{c}\text { Median } \\
\text { (IQR) } \\
70(62.8-75.0)\end{array}$ & $\begin{array}{c}\text { Median } \\
\text { (IQR) } \\
71(66.0-75.0)\end{array}$ & $\begin{array}{c}\text { Median } \\
\text { (IQR) } \\
13.7(\text { (9.3-25.0) }\end{array}$ & $\begin{array}{c}\text { Median } \\
(\text { IQR) } \\
10.2(6.7-17.7)\end{array}$ & NA & NA \\
\hline Martini et al. (18) & 2019 & Italy & 2011-2017 & 285 & 1,472 & $\begin{array}{c}\text { Median } \\
\text { (IQR) } \\
64.8(58.9-70.0)\end{array}$ & $\begin{array}{c}\text { Median } \\
\text { (IQR) } \\
64.6(59.0-69.7)\end{array}$ & $\begin{array}{c}\text { Median } \\
\text { (IQR) } \\
7.2(5.5-10.6)\end{array}$ & $\begin{array}{c}\text { Median } \\
\text { (IQR) } \\
6.3(4.6-8.3)\end{array}$ & $\begin{array}{l}\text { Median } \\
30\end{array}$ & $\begin{array}{l}\text { Median } \\
30\end{array}$ \\
\hline Hou et al. (19) & 2019 & China & $2007-2017$ & 94 & 226 & $\begin{array}{c}\text { Median } \\
\text { (IQR) } \\
67.9(45-80)\end{array}$ & $\begin{array}{c}\text { Median } \\
\text { (IQR) } \\
67.9(45-80)\end{array}$ & $\begin{array}{c}\text { Median } \\
\text { (IQR) } \\
14.4(1-123)\end{array}$ & $\begin{array}{c}\text { Median } \\
(\text { IQR) } \\
14.4(1-123)\end{array}$ & NA & NA \\
\hline Herforth et al. (20) & 2018 & USA & 1988-2015 & 1,902 & 2,063 & $\begin{array}{c}\text { Median } \\
\text { (IQR) } \\
62(58-66)\end{array}$ & $\begin{array}{c}\text { Median } \\
\text { (IQR) } \\
63(58-67)\end{array}$ & $\begin{array}{l}\text { Median (IQR) } \\
7.5(5.2-12)\end{array}$ & $\begin{array}{l}\text { Median (IQR) } \\
5.9(4.4-8.5)\end{array}$ & $\begin{array}{l}\text { Median (IQR) } \\
93 \text { (53-152) }\end{array}$ & $\begin{array}{l}\text { Median (IQR) } \\
105(63-147)\end{array}$ \\
\hline Tatsugami et al. (21) & 2017 & Japan & 2009-2013 & 594 & 1,794 & $\begin{array}{c}\text { Mean } \pm \text { SD } \\
64.9 \pm 6.2\end{array}$ & $\begin{array}{c}\text { Mean } \pm \text { SD } \\
65.3 \pm 6.2\end{array}$ & $\begin{array}{l}\text { Median (range) } \\
6.6(1.8-57.1)\end{array}$ & $\begin{array}{l}\text { Median (range) } \\
7.7(3.0-69.8)\end{array}$ & $\begin{array}{c}\text { Median (range) } \\
9(1-83)\end{array}$ & $\begin{array}{l}\text { Median } \\
\text { (range) } \\
9(1-83)\end{array}$ \\
\hline Seo et al. (8) & 2017 & Korea & 2008-2014 & 50 & 94 & $\begin{array}{c}\text { Mean } \pm \text { SD } \\
64.6 \pm 6.5\end{array}$ & $\begin{array}{l}\text { Mean } \pm \text { SD } \\
67.3 \pm 6.7\end{array}$ & $\begin{array}{l}\text { Mean } \pm \text { SD } \\
16.3 \pm 11.4\end{array}$ & $\begin{array}{c}\text { Mean } \pm \text { SD } \\
10.5 \pm 6.7\end{array}$ & $\begin{array}{c}\text { Mean } \pm \text { SD } \\
55.4 \pm 3.9\end{array}$ & $\begin{array}{l}\text { Mean } \pm \text { SD } \\
64.1 \pm 2.0\end{array}$ \\
\hline Meyer et al. (22) & 2017 & USA & 1992-2005 & 118 & 785 & $\begin{array}{c}\text { Median } \\
\text { (IQR) } \\
63(60-67)\end{array}$ & $\begin{array}{c}\text { Median } \\
\text { (IQR) } \\
63(58-66)\end{array}$ & $\begin{array}{l}\text { Median (IQR) } \\
6(4.3-9.0)\end{array}$ & $\begin{array}{c}\text { Median } \\
(\text { IQR) } \\
6.4(4.6-8.9)\end{array}$ & $\begin{array}{c}\text { Median } \\
\text { (IQR) } \\
132(86-145)\end{array}$ & $\begin{array}{c}\text { Median } \\
(\text { IQR }) \\
133(99-157)\end{array}$ \\
\hline Abdollah et al. (23) & 2016 & MC & 2002-2013 & 1,045 & 11,470 & $\begin{array}{c}\text { Median (IQR) } \\
62(56-67)\end{array}$ & $\begin{array}{c}\text { Median (IQR) } \\
61 \text { (55-56) }\end{array}$ & $\begin{array}{l}\text { Median (IQR) } \\
6.2(4.7-9.6)\end{array}$ & $\begin{array}{c}\text { Median } \\
\quad(\text { IQR) } \\
5.2(4.1-7.2)\end{array}$ & $\begin{array}{l}\text { Median } \\
39\end{array}$ & $\begin{array}{l}\text { Median } \\
39\end{array}$ \\
\hline Whalen et al. (24) & 2015 & USA & 2005-2011 & 126 & 453 & $\begin{array}{l}\text { Mean } \pm \text { SD } \\
61.0 \pm 7.7\end{array}$ & $\begin{array}{c}\text { Mean } \pm \text { SD } \\
61.3 \pm 7.0\end{array}$ & $\begin{array}{c}\text { Mean } \pm \text { SD } \\
9.2 \pm 8.6\end{array}$ & $\begin{array}{c}\text { Mean } \pm \text { SD } \\
6.1 \pm 5.4\end{array}$ & $\begin{array}{c}\text { Median (range) } \\
20.5(1-80)\end{array}$ & $\begin{array}{c}\text { Median } \\
\text { (range) } \\
20.5(1-80)\end{array}$ \\
\hline Retèl et al. (25) & 2014 & Switzerland & 1990-2008 & 479 & 775 & $\begin{array}{l}\text { Mean } \pm \text { SD } \\
63.4 \pm 6.0\end{array}$ & $\begin{array}{l}\text { Mean } \pm \text { SD } \\
62.9 \pm 6.5\end{array}$ & NA & NA & $\begin{array}{l}\text { Median (range) } \\
73.2(2-120)\end{array}$ & $\begin{array}{c}\text { Median } \\
\text { (range) } \\
73.2(2-120)\end{array}$ \\
\hline Rouanne et al. (26) & 2014 & France & 1988-2001 & 108 & 295 & $\begin{array}{l}\text { Median (range) } \\
66(47-77)\end{array}$ & $\begin{array}{c}\text { Median (range) } \\
66(46-81)\end{array}$ & $\begin{array}{l}\text { Median (range) } \\
10(2-158)\end{array}$ & $\begin{array}{c}\text { Median (range) } \\
10(0.5-134)\end{array}$ & $\begin{array}{l}\text { Median (range) } \\
139 \text { (126-231) }\end{array}$ & $\begin{array}{c}\text { Median } \\
\text { (range) } \\
147(134-251)\end{array}$ \\
\hline Sammon et al. (27) & 2013 & USA & 1993-2010 & 162 & 632 & $\begin{array}{c}\text { Mean } \pm \text { SD } \\
63.1 \pm 8.9\end{array}$ & $\begin{array}{c}\text { Mean } \pm \text { SD } \\
63.5 \pm 7.8\end{array}$ & $\begin{array}{c}\text { Mean } \pm \text { SD } \\
6.9 \pm 4.6\end{array}$ & $\begin{array}{c}\text { Mean } \pm \text { SD } \\
5.3 \pm 3.3\end{array}$ & $\begin{array}{l}\text { Median (IQR) } \\
54(27-84)\end{array}$ & $\begin{array}{c}\text { Median (IQR) } \\
54(27-84)\end{array}$ \\
\hline Lee et al. (28) & 2013 & Korea & 2005-2011 & 167 & 200 & $\begin{array}{l}\text { Mean } \pm \text { SD } \\
67.9 \pm 5.7\end{array}$ & $\begin{array}{c}\text { Mean } \pm \text { SD } \\
67.8 \pm 5.3\end{array}$ & $\begin{array}{l}\text { Mean } \pm \text { SD } \\
11.2 \pm 10.4\end{array}$ & $\begin{array}{c}\text { Mean } \pm \text { SD } \\
8.4 \pm 6.4\end{array}$ & NA & NA \\
\hline Hashimoto et al. (29) & 2013 & Japan & 2006-2011 & 54 & 190 & $\begin{array}{c}\text { Mean } \pm \text { SD } \\
64.8 \pm 5.7\end{array}$ & $\begin{array}{c}\text { Mean } \pm \text { SD } \\
64.0 \pm 6.8\end{array}$ & $\begin{array}{l}\text { Mean } \pm \text { SD } \\
12.5 \pm 12.6\end{array}$ & $\begin{array}{c}\text { Mean } \pm \text { SD } \\
9.3 \pm 7.3\end{array}$ & NA & NA \\
\hline Abdollah et al. (30) & 2013 & Italy & 1998-2010 & 305 & 1,198 & $\begin{array}{c}\text { Median (range) } \\
64.6(40.5-81.1)\end{array}$ & $\begin{array}{c}\text { Median } \\
\text { (range) } \\
64.8(42.3-82.2)\end{array}$ & $\begin{array}{c}\text { Median (range) } \\
6.6(1-74.1)\end{array}$ & $\begin{array}{l}\text { Median (range) } \\
6.2(0.2-47.8)\end{array}$ & $\begin{array}{l}\text { Mean } \\
122.5\end{array}$ & $\begin{array}{l}\text { Mean } \\
122.5\end{array}$ \\
\hline
\end{tabular}


TABLE 1 | Continued

\begin{tabular}{|c|c|c|c|c|c|c|c|c|c|c|c|}
\hline \multirow[t]{2}{*}{ Author } & \multirow[t]{2}{*}{ Year } & \multirow[t]{2}{*}{ Country } & \multirow{2}{*}{$\begin{array}{l}\text { Recruitment } \\
\text { period }\end{array}$} & \multicolumn{2}{|c|}{ No. of patients } & \multicolumn{2}{|c|}{ Age (years) } & \multicolumn{2}{|c|}{ Pre-PSA } & \multicolumn{2}{|c|}{ Follow-up (months) } \\
\hline & & & & PSMs & NSMs & PSMs & NSMs & PSMs & NSMs & PSMs & NSMs \\
\hline Savdie et al. (31) & 2012 & Australia & 1997-2003 & 285 & 655 & $\begin{array}{l}\text { Median (range) } \\
61.7(46.4-81)\end{array}$ & $\begin{array}{c}\text { Median } \\
\text { (range) } \\
61.2(42.2-77.4)\end{array}$ & $\begin{array}{c}\text { Median (range) } \\
8.7(2-63)\end{array}$ & $\begin{array}{c}\text { Median (range) } \\
7.5(0.4-84)\end{array}$ & $\begin{array}{l}\text { Median (range) } \\
82(5-146)\end{array}$ & $\begin{array}{c}\text { Median } \\
\text { (range) } \\
82(5-146)\end{array}$ \\
\hline Lu et al. (32) & 2012 & China & 1993-1999 & 250 & 544 & $\begin{array}{c}\text { Median } \\
\text { (IQR) } \\
62(57-66)\end{array}$ & $\begin{array}{c}\text { Median } \\
\text { (IQR) } \\
62(52-66)\end{array}$ & $\begin{array}{l}\text { Median (IQR) } \\
6.2(4.5-9.3)\end{array}$ & $\begin{array}{c}\text { Median } \\
(\text { IQR) } \\
5.9(4.5-8.0)\end{array}$ & $\begin{array}{c}\text { Median } \\
\text { (IQR) } \\
115.2(72-132)\end{array}$ & $\begin{array}{c}\text { Median } \\
(\text { IQR) } \\
120(78-135.6)\end{array}$ \\
\hline Karavitakis et al. (33) & 2012 & UK & 2007-2009 & 31 & 64 & $\begin{array}{l}\text { Mean } \\
62.9\end{array}$ & $\begin{array}{l}\text { Mean } \\
61.3\end{array}$ & $\begin{array}{c}\text { Mean } \\
13.9\end{array}$ & $\begin{array}{c}\text { Mean } \\
10.9\end{array}$ & NA & NA \\
\hline Corcoran et al. (34) & 2012 & Australia & 1995-2010 & 370 & 1,144 & $\begin{array}{c}\text { Median (range) } \\
61.5(40.2-79.8)\end{array}$ & $\begin{array}{c}\text { Median } \\
\text { (range) } \\
61.5(40.2-79.8)\end{array}$ & $\begin{array}{c}\text { Mean } \pm \text { SD } \\
7.8 \pm 6.6\end{array}$ & $\begin{array}{c}\text { Mean } \pm \text { SD } \\
7.8 \pm 6.6\end{array}$ & $\begin{array}{l}\text { Median (range) } \\
22.2(0.8-181)\end{array}$ & $\begin{array}{c}\text { Median } \\
\text { (range) } \\
22.2(0.8-181)\end{array}$ \\
\hline Li et al. (35) & 2011 & China & 2000-2009 & 57 & 92 & $\begin{array}{c}\text { Mean } \pm \text { SD } \\
70.2 \pm 6.3\end{array}$ & $\begin{array}{c}\text { Mean } \pm S D \\
69.0 \pm 6.0\end{array}$ & $\begin{array}{l}\text { Mean } \pm \text { SD } \\
13.4 \pm 17.6\end{array}$ & $\begin{array}{c}\text { Mean } \pm \text { SD } \\
8.0 \pm 5.8\end{array}$ & $\begin{array}{l}\text { Mean } \pm \text { SD } \\
46.8 \pm 27.8\end{array}$ & $\begin{array}{l}\text { Mean } \pm \text { SD } \\
46.8 \pm 27.8\end{array}$ \\
\hline Coelho et al. (36) & 2010 & USA & 2008-2009 & 101 & 775 & $\begin{array}{c}\text { Median } \\
\text { (IQR) } \\
62(56-66)\end{array}$ & $\begin{array}{c}\text { Median } \\
\text { (IQR) } \\
61(56-66)\end{array}$ & $\begin{array}{c}\text { Median } \\
(\text { IQR) } \\
5(3.9-6.9)\end{array}$ & $\begin{array}{c}\text { Median } \\
(\text { IQR) } \\
4.9(3.8-6.6)\end{array}$ & NA & NA \\
\hline Boorjian et al. (37) & 2010 & USA & 1990-2006 & 3,651 & 8,078 & $\begin{array}{c}\text { Median } \\
\text { (IQR) } \\
64 \text { (59-69) }\end{array}$ & $\begin{array}{c}\text { Median } \\
\text { (IQR) } \\
63(57-68)\end{array}$ & $\begin{array}{c}\text { Median } \\
\text { (IQR) } \\
8.1(5.4-14.1)\end{array}$ & $\begin{array}{c}\text { Median } \\
(\text { IQR) } \\
5.9(4.1-8.7)\end{array}$ & $\begin{array}{c}\text { Median } \\
\text { (IQR) } \\
98.4(52.8-145.2)\end{array}$ & $\begin{array}{c}\text { Median } \\
(\text { IQR }) \\
98.4(52.8-145.2)\end{array}$ \\
\hline Alkhateeb et al. (38) & 2010 & Canada & 1992-2008 & 264 & 1,004 & $\begin{array}{c}\text { Mean } \pm \text { SD } \\
62 \pm 6.6\end{array}$ & $\begin{array}{c}\text { Mean } \pm \text { SD } \\
62 \pm 6.6\end{array}$ & $\begin{array}{c}\text { Mean } \\
\text { (range) } \\
7.7(0.1-65.9)\end{array}$ & $\begin{array}{c}\text { Mean } \\
\text { (range) } \\
7.7(0.1-65.9)\end{array}$ & $\begin{array}{c}\text { Mean } \\
\text { (range) } \\
78.1(3-192)\end{array}$ & $\begin{array}{c}\text { Mean } \\
\text { (range) } \\
78.1(3-192)\end{array}$ \\
\hline Shikanov et al. (39) & 2009 & USA & 2003-2008 & 243 & 1,155 & $\begin{array}{c}\text { Median } \\
(\text { IQR) } \\
59(54-65)\end{array}$ & $\begin{array}{c}\text { Median } \\
\text { (IQR) } \\
60(55-65)\end{array}$ & $\begin{array}{c}\text { Median } \\
\text { (IQR) } \\
5.6(4.4-8.1)\end{array}$ & $\begin{array}{c}\text { Median } \\
\text { (IQR) } \\
5.1(4.1-7.1)\end{array}$ & $\begin{array}{c}\text { Median } \\
\text { (IQR) } \\
12.3(6.3-18.9)\end{array}$ & $\begin{array}{c}\text { Median } \\
(\text { IQR }) \\
12.3(6.3-20.1)\end{array}$ \\
\hline Ficarra et al. (40) & 2009 & Italy & 2005-2008 & 95 & 227 & $\begin{array}{l}\text { Mean } \\
61.4\end{array}$ & $\begin{array}{l}\text { Mean } \\
61.4\end{array}$ & NA & NA & $\begin{array}{l}\text { Median } \\
14\end{array}$ & $\begin{array}{l}\text { Median } \\
14\end{array}$ \\
\hline
\end{tabular}

SD, standard deviation; NA, data not applicable; MC, Multi-centers; PSMs, positive surgical margins; NSMs, negative surgical margins. 
TABLE 2 | The main pathological characteristics of all studies included in this meta-analysis.

\begin{tabular}{|c|c|c|c|c|c|c|c|c|}
\hline \multirow[t]{2}{*}{ Author } & \multirow[t]{2}{*}{ Stagingsystem } & \multirow[t]{2}{*}{ Gradingsystem } & \multicolumn{2}{|c|}{ Biopsy GS $<6 / \geq 7$} & \multicolumn{2}{|c|}{ Pathological GS $<6 / \geq 7$} & \multicolumn{2}{|c|}{ Pathological stage $1-2 / 3-4$} \\
\hline & & & PSMs & NSMs & PSMs & NSMs & PSMs & NSMs \\
\hline Celik et al. (15) & TNM & 2014 ISUP & NA & NA & NA & NA & $427 / 466$ & $1,377 / 413$ \\
\hline Porcaro et al. (16) & 2010 TNM & 2014 ISUP & $81 / 111$ & $262 / 278$ & $19 / 173$ & $107 / 433$ & $161 / 31$ & $453 / 87$ \\
\hline Tian et al. (17) & 2012TNM & Gleason score & NA & NA & NA & NA & $75 / 67$ & $212 / 64$ \\
\hline Martini et al. (18) & TNM & Gleason score & NA & NA & $203 / 82$ & $1,246 / 208$ & 108/177 & $969 / 503$ \\
\hline Hou et al. (19) & TNM & Gleason score & $27 / 67$ & $101 / 125$ & $16 / 78$ & $84 / 142$ & $46 / 48$ & $174 / 52$ \\
\hline Herforth et al. (20) & TNM & Gleason score & NA & NA & NA & NA & $1,249 / 653$ & $1,567 / 496$ \\
\hline Tatsugami et al. (21) & TNM & Gleason score & $172 / 422$ & $1,200 / 594$ & $46 / 548$ & $276 / 1,518$ & $539 / 55$ & 62/594 \\
\hline Seo et al. (8) & TNM & Gleason score & 14/36 & $40 / 54$ & NA & NA & $34 / 16$ & $84 / 10$ \\
\hline Meyer et al. (22) & 2002TNM & Gleason score & $98 / 20$ & $625 / 120$ & $69 / 49$ & $510 / 275$ & NA & NA \\
\hline Abdollah et al. (23) & TNM & Gleason score & 436/891 & $1,726 / 2,237$ & 138/1,198 & $1,167 / 2,796$ & $373 / 954$ & $2,883 / 1,080$ \\
\hline Whalen et al. (24) & 1997TNM & Gleason score & 30/96 & $214 / 239$ & $30 / 96$ & 214/239 & $51 / 75$ & $365 / 88$ \\
\hline Retèl et al. (25) & TNM & Gleason score & NA & NA & $224 / 255$ & $502 / 273$ & $239 / 240$ & $629 / 146$ \\
\hline Rouanne et al. (26) & TNM & Gleason score & $81 / 27$ & $233 / 62$ & $49 / 59$ & $181 / 114$ & $35 / 73$ & $224 / 71$ \\
\hline Sammon et al. (27) & TNM & Gleason score & NA & NA & 67/95 & $525 / 107$ & $47 / 115$ & $298 / 334$ \\
\hline Lee et al. (28) & TNM & Gleason score & NA & NA & $30 / 136$ & $69 / 131$ & $88 / 79$ & $169 / 31$ \\
\hline Hashimoto et al. (29) & NA & Gleason score & $18 / 36$ & $63 / 127$ & NA & NA & NA & NA \\
\hline Abdollah et al. (30) & TNM & Gleason score & NA & NA & $115 / 190$ & $635 / 563$ & $256 / 49$ & $1,115 / 83$ \\
\hline Savdie et al. (31) & TNM & Gleason score & NA & NA & $75 / 210$ & $241 / 414$ & $105 / 180$ & $438 / 217$ \\
\hline Lu et al. (32) & TNM & Gleason score & NA & NA & $80 / 170$ & $293 / 251$ & $161 / 89$ & $468 / 76$ \\
\hline Karavitakis et al. (33) & TNM & Gleason score & $18 / 13$ & $43 / 21$ & $7 / 21$ & 22/43 & $14 / 17$ & 45/19 \\
\hline Corcoran et al. (34) & TNM & Gleason score & NA & NA & $47 / 323$ & $290 / 854$ & 182/188 & $924 / 220$ \\
\hline Li et al. (35) & 1992TNM & Gleason score & NA & NA & NA & NA & NA & NA \\
\hline Coelho et al. (36) & TNM & Gleason score & $56 / 45$ & 453/322 & $21 / 80$ & $310 / 463$ & $43 / 58$ & $669 / 106$ \\
\hline Boorjian et al. (37) & TNM & Gleason score & $1,905 / 1,125$ & $5,372 / 1,621$ & $1,806 / 1,839$ & $5,719 / 2,328$ & 2,072/1,579 & $6,767 / 1,289$ \\
\hline Alkhateeb et al. (38) & TNM & Gleason score & NA & NA & 42/222 & 310/694 & $116 / 148$ & $737 / 267$ \\
\hline Shikanov et al. (39) & TNM & Gleason score & $118 / 125$ & $727 / 428$ & $73 / 170$ & $592 / 563$ & $120 / 123$ & $980 / 175$ \\
\hline Ficarra et al. (40) & 2002TNM & Gleason score & $67 / 28$ & $187 / 40$ & 26/69 & $112 / 115$ & $21 / 74$ & $177 / 50$ \\
\hline
\end{tabular}

NA, data not applicable; PSMs, positive surgical margins; NSMs, negative surgical margins; GS, Gleason Score; ISUP, International Society of Urologic Pathology (ISUP) system.

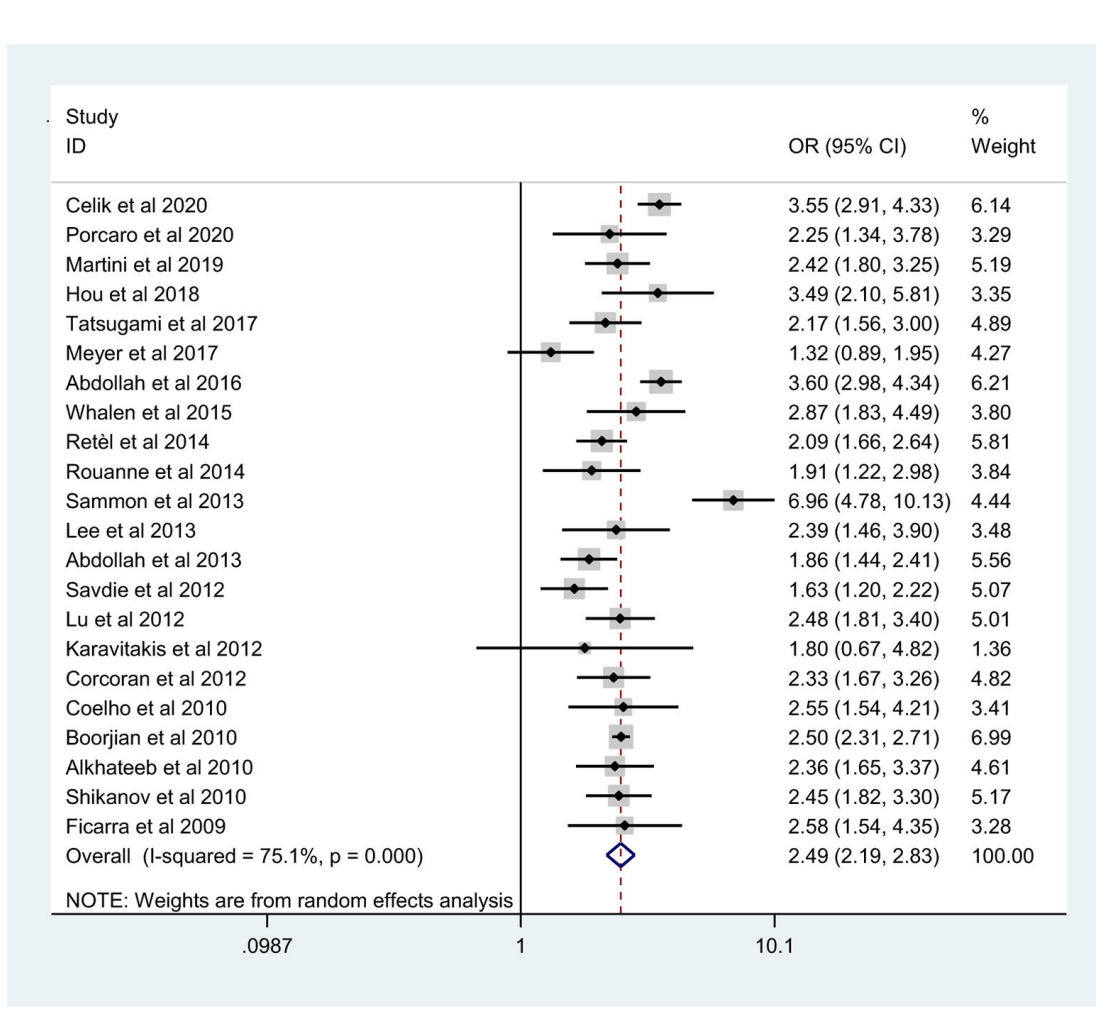

FIGURE 2 | Forest plot for the association between pathological GS and PSMs risk. 


\section{Meta-Analysis}

The pooled results from the included studies indicated that PSMs were associated with pathological GS $(<6 / \geq 7)$ (RE model, pooled $\mathrm{OR}=2.49 ; 95 \% \mathrm{CI}: 2.19-2.83 ; \mathrm{P}<0.001$, Figure 2), pathological stage $(<\mathrm{T} 2 / \geq \mathrm{T} 3)$ (RE model, pooled $\mathrm{OR}=3.90$; 95\% CI: 3.18-4.79; $\mathrm{P}<0.001$, Figure 3), biopsy GS $(<6 / \geq 7)$ (RE model, pooled $\mathrm{OR}=$ 1.53; 95\% CI: 1.31-1.79; P < 0.001, Figure 4), p-PSA (FE model, pooled $\mathrm{SMD}=0.37 ; 95 \% \mathrm{CI}: 0.31-0.43 ; \mathrm{P}<0.001$, Figure 5A), positive lymph node (PLN) (RE model, pooled OR $=3.12 ; 95 \% \mathrm{CI}$ : 2.28-4.27; $\mathrm{P}<0.001$, Figure 5B), extraprostatic extension (EPE) (RE model, pooled $\mathrm{OR}=4.44$; 95\% CI: 3.25-6.09; $\mathrm{P}<0.001$, Figure 5C), and seminal vesicle invasion (SVI) (RE model, pooled $\mathrm{OR}=4.19$; 95\% CI: 2.87-6.13; $\mathrm{P}<0.001$, Figure 5D).

The results of meta-analysis of PSMs showed that no significant associations were found between PSMs and age (RE model, pooled $\mathrm{SMD}=0.01 ; 95 \% \mathrm{CI}:-0.07-0.10 ; \mathrm{P}=0.735$, Figure 6A), nerve sparing (RE model, pooled $\mathrm{OR}=0.90$; 95\% CI: $0.71-1.14 ; \mathrm{P}=0.388$, Figure 6B), body mass index (BMI) (RE model, pooled SMD $=0.12 ; 95 \% \mathrm{CI}:-0.05-0.30 ; \mathrm{P}=0.162$, Figure 6C), and prostate volume (RE model, pooled $\mathrm{SMD}=$ -0.28 ; 95\% CI: $-0.62-0.05$; $\mathrm{P}=0.097$, Figure 6D).

\section{Subgroup Analysis}

Considering that there was no significant heterogeneity in p-PSA and the number of studies that evaluated BMI, SVI, and prostate volume was relatively small, we only conducted subgroup analysis for biopsy GS, pathological GS, pathological stage, PLN, EPE, age, and nerve sparing (Table 3). Subgroup analyses were conducted according to the geographical region (Asian vs. non-Asian), year of publication ( $\geq 2014$ vs. <2014), number of patients $(\geq 1,000 v s .<1,000)$, and median follow-up ( $\geq 70$ months $v s .<70$ months). The results of subgroup analysis were roughly the same as overall results. Besides, the heterogeneity decreased significantly in some subgroup analyses, such as geographical region in Asian, year of publication $<2014$, and number of patients $<1,000$ cases.

\section{Sensitivity Analysis}

To validate the reliability of our results, sensitivity analysis was performed. As shown in Supplementary Figure S1, the combined ORs for biopsy GS ranged from 1.47 (95\% CI: 1.25 -1.72 ) to 1.58 (95\% CI: 1.37-1.85) (Supplementary Figure S1A), the combined ORs for pathological GS ranged from 2.39 (95\%

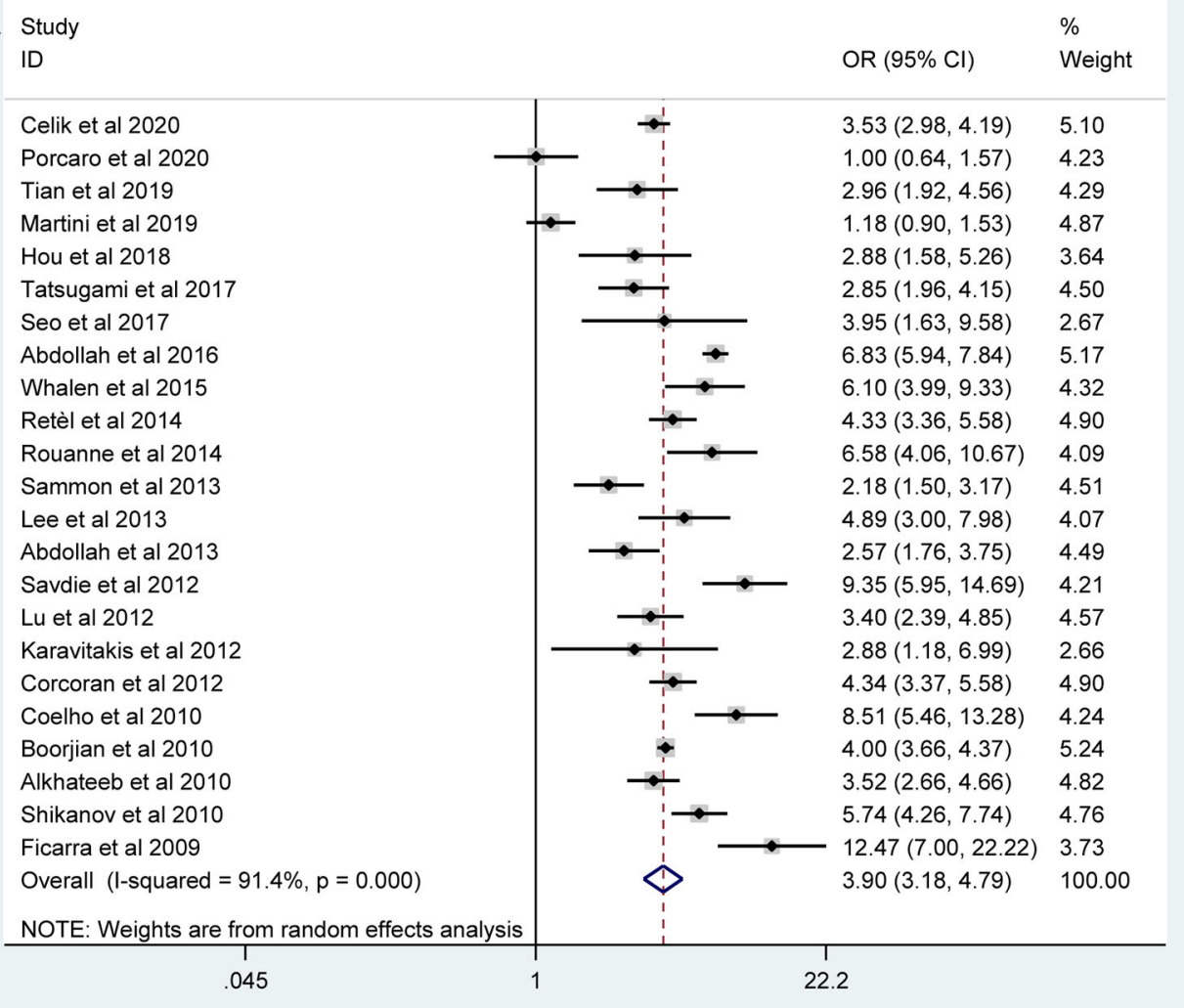

FIGURE 3 | Forest plot reflecting the association between pathological stage and PSMs. 


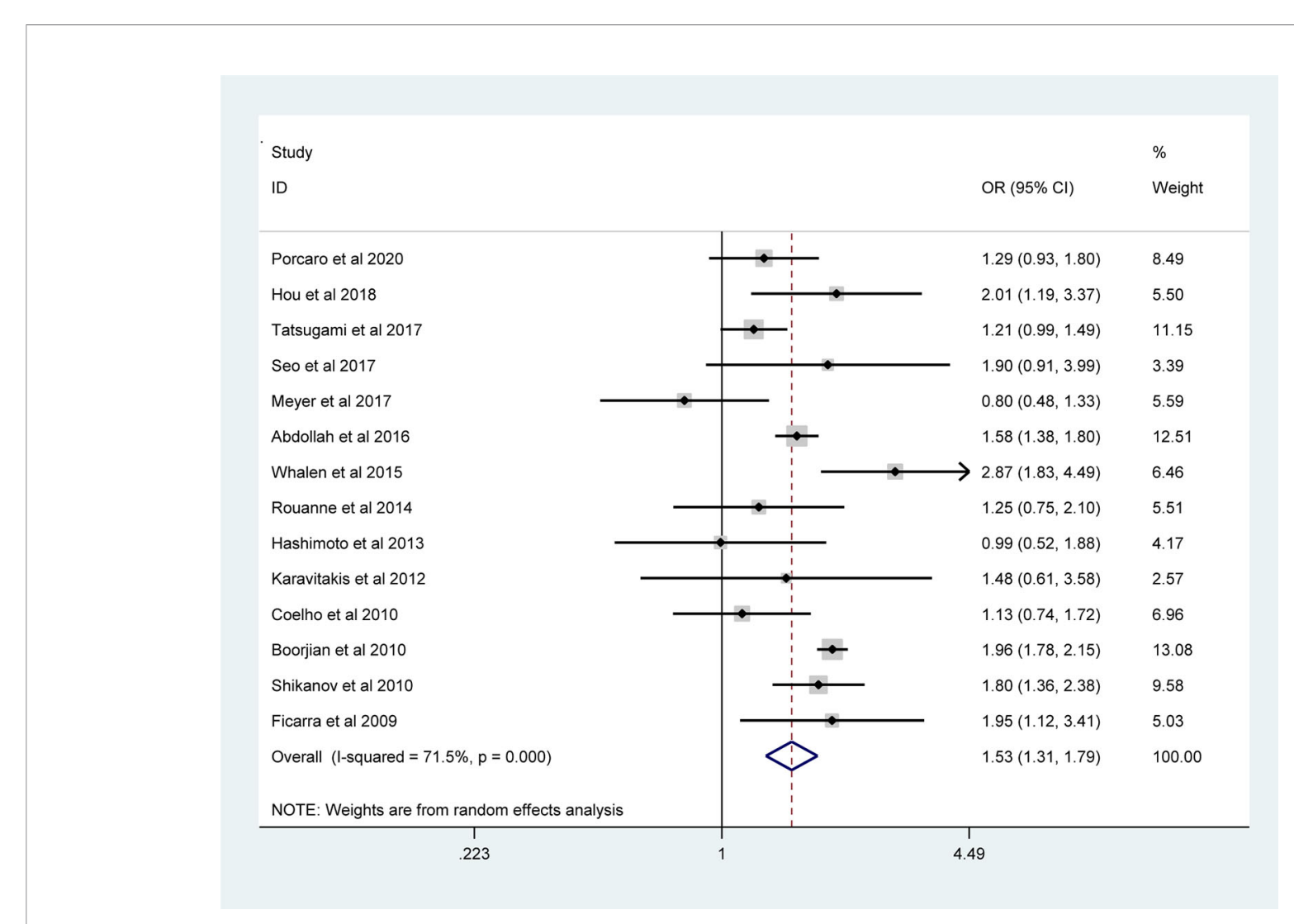

FIGURE 4 | Forest plot assessing the correlation of biopsy GS and PSMs.

CI: 2.14-2.67) to 2.56 (95\% CI: 2.26-2.90) (Supplementary Figure S1B), the combined ORs for pathological stage ranged from 3.73 (95\% CI: 3.04-4.58) to 4.15 (95\% CI: 3.47-4.96) (Supplementary Figure S1C), the combined ORs for PLN ranged from 2.88 (95\% CI: $2.08-4.00)$ to 3.51 (95\% CI: $2.67-$ 4.79) (Supplementary Figure S1D), the combined ORs for nerve sparing ranged from 0.83 (95\% CI: $0.66-1.04)$ to 0.97 (95\% CI: 0.74-1.27) (Supplementary Figure S1E), and the combined ORs for EPE ranged from 3.84 (95\% CI: 3.05-4.85) to 4.68 (95\% CI: 3.36-6.53) (Supplementary Figure S1F). The pooled SMD for pPSA ranged from 0.36 (95\% CI: 0.29-0.42) to 0.44 (95\% CI: $0.35-$ 0.54) (Supplementary Figure S2A), and the pooled SMD for age ranged from -0.01 (95\% CI: $-0.09-0.07)$ to 0.03 (95\% CI: $-0.05-$ 0.12) (Supplementary Figure S2B). These data suggested that the results were statistically robust. Because the number of included studies for BMI, EPE, SVI, and prostate volume were small, the sensitivity analysis was not valuable.

\section{Publication Bias}

The shape of funnel plots did not reveal any evidence of asymmetry (Figure 7). The statistical results of Egger's test still did not show any publication bias for biopsy GS (p- Egger = 0.277 , Figure 7A), pathological GS ( $\mathrm{p}$ - Egger $=0.945$, Figure 7B), pathological stage (p- Egger $=0.830$, Figure 7C), PLN (pEgger $=0.605$, Figure 7D), EPE $(p-$ Egger $=0.513$, Figure 7E), SVI $(\mathrm{p}$ - Egger $=0.797$, Figure $7 F)$, age $(\mathrm{p}$ - Egger $=0.431$, Figure
7G), and nerve sparing ( $\mathrm{p}$ - Egger $=0.197$, Figure $7 \mathbf{H})$. However, a minimal publication bias existed in p-PSA ( $\mathrm{p}$ - Egger $=0.047$ ). As the number of studies on prostate volume and BMI was limited, the publication bias was not assessed.

\section{DISCUSSION}

PSMs are unfavorable pathological features, which suggest incomplete tumor resection and confer poorer cancer control after RP (38). It was reported that PSMs were present in 11-38\% of patients treated by RP and patients with PSMs have a higher risk of BCR compared to those with negative surgical margins (NSMs) (41). A multi-institutional review in 2009 conducted by Yossepowitch et al. (42) concluded that PSMs in RP specimens may be considered as an adverse outcome following RP. Consistent with these findings, our recent studies $(6,7)$ demonstrated the adverse effect of PSMs on both BCR and cancer-specific survival through a systematic review and metaanalysis. However, not all patients with PSMs have poor tumor outcomes, and some patients with localized PCa will show tumor progression even in the no-PSMs cases.

PSMs are factors that may be modified by the surgical technique. It seems that surgeon's experience plays an important role in the decrease in the incidence of PSMs (43). Considerable efforts have been devoted to identifying factors, 


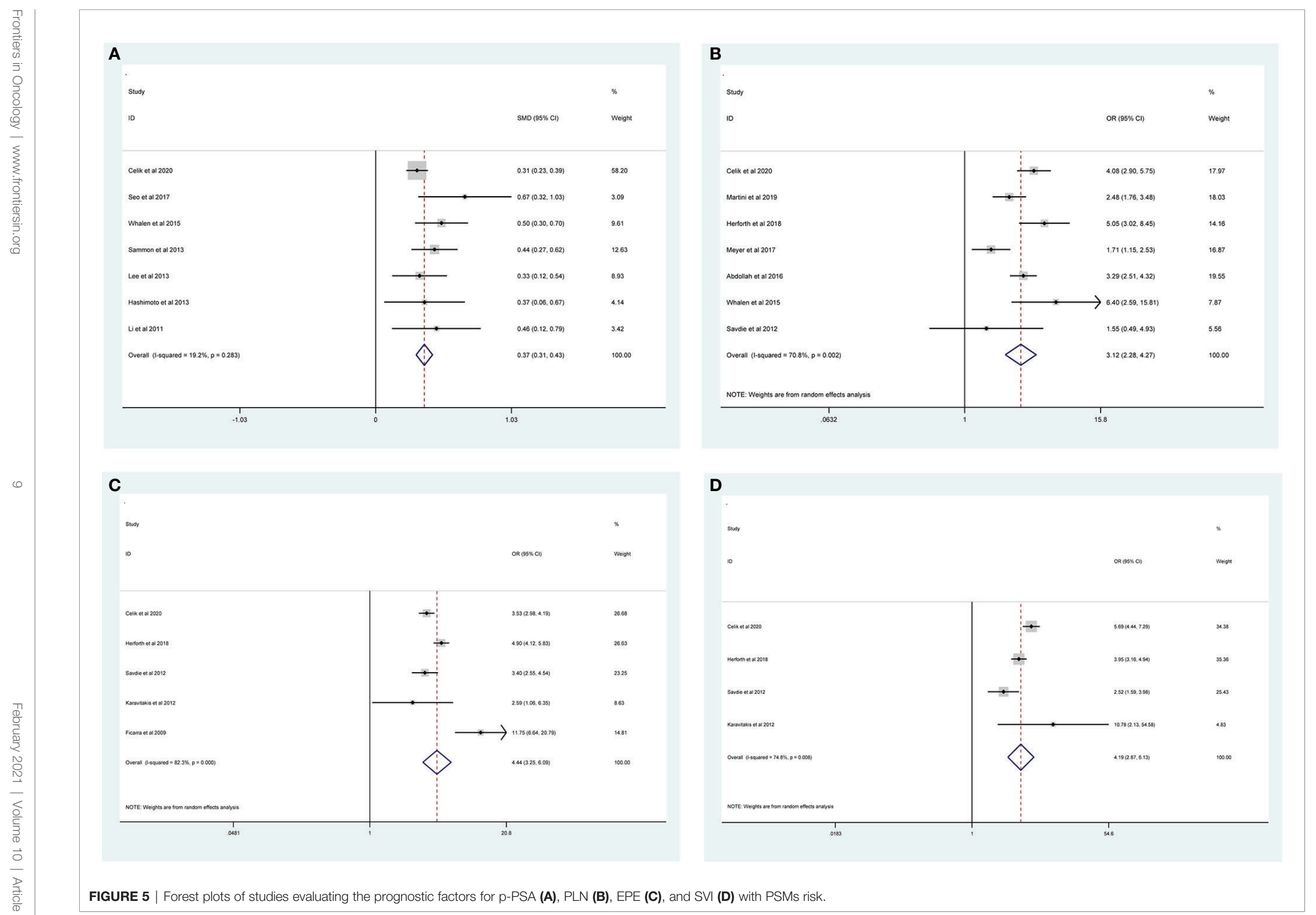




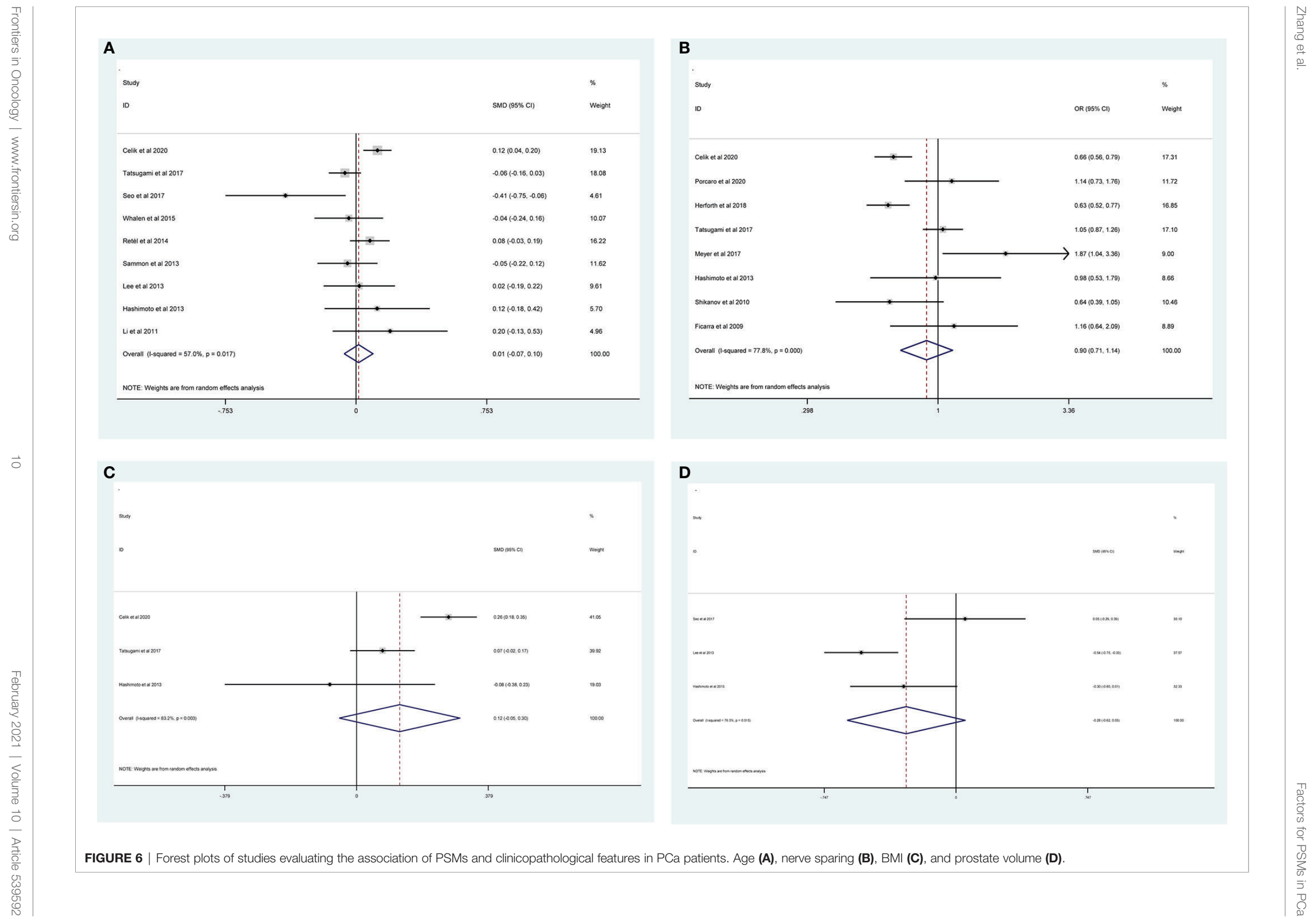


TABLE 3 | Summary and subgroup results for PSMs and clinicopathological features in PCa patients.

\begin{tabular}{|c|c|c|c|c|c|c|}
\hline \multirow[t]{2}{*}{ Analysis specification } & \multirow[t]{2}{*}{ No. of studies } & \multicolumn{2}{|c|}{ Study heterogeneity } & \multirow[t]{2}{*}{ Effects model } & \multirow[t]{2}{*}{ Pooled OR/SMD (95\% Cl) } & \multirow[t]{2}{*}{ P-Value } \\
\hline & & $I^{2}(\%)$ & $\mathbf{P}_{\text {heterogeneity }}$ & & & \\
\hline \multicolumn{7}{|l|}{ BMI } \\
\hline Overall & 3 & 83.2 & 0.003 & Random & $0.12(-0.05,0.30)$ & 0.162 \\
\hline \multicolumn{7}{|l|}{ p-PSA } \\
\hline Overall & 7 & 19.2 & 0.283 & Fixed & $0.37(0.31,0.43)$ & $<0.001$ \\
\hline \multicolumn{7}{|l|}{ SVI } \\
\hline Overall & 4 & 74.8 & 0.008 & Random & $4.19(2.87,6.13)$ & $<0.001$ \\
\hline \multicolumn{7}{|l|}{ Prostate volume } \\
\hline Overall & 3 & 76.3 & 0.015 & Random & $-0.28(-0.62,0.05)$ & 0.097 \\
\hline \multicolumn{7}{|l|}{ Age } \\
\hline Overall & 9 & 57 & 0.017 & Random & $0.01(-0.07,0.10)$ & 0.735 \\
\hline \multicolumn{7}{|l|}{ Geographical region } \\
\hline Asian & 5 & 49.6 & 0.094 & Random & $-0.03(-0.17,0.12)$ & 0.724 \\
\hline non-Asian & 4 & 36.4 & 0.193 & Fixed & $0.06(-0.02,0.14)$ & 0.149 \\
\hline \multicolumn{7}{|l|}{ Year of publication } \\
\hline$\geq 2014$ & 5 & 75.6 & 0.003 & Random & $-0.01(-0.12,0.11)$ & 0.916 \\
\hline$<2014$ & 4 & 0 & 0.543 & Fixed & $0.02(-0.09,0.14)$ & 0.675 \\
\hline \multicolumn{7}{|l|}{ No. of patients } \\
\hline$\geq 1,000$ & 3 & 78.4 & 0.010 & Random & $0.05(-0.07,0.16)$ & 0.442 \\
\hline$<1,000$ & 6 & 34.0 & 0.182 & Fixed & $-0.02(-0.14,0.10)$ & 0.719 \\
\hline Biopsy GS $(<6 / \geq 7)$ & & & & & & \\
\hline Overall & 14 & 71.5 & $<0.001$ & Random & $1.53(1.31,1.79)$ & $<0.001$ \\
\hline Geographical region & & & & & & \\
\hline Asian & 4 & 28.2 & 0.243 & Fixed & $1.19(0.90,1.58)$ & 0.227 \\
\hline non-Asian & 10 & 64.1 & 0.003 & Random & 1.65 (1.42,1.93) & $<0.001$ \\
\hline Year of publication & & & & & & \\
\hline$\geq 2014$ & 9 & 64.8 & 0.004 & Random & $1.44(1.17,1.76)$ & $<0.001$ \\
\hline$<2014$ & 5 & 41.1 & 0.147 & Fixed & $1.75(1.44,2.11)$ & $<0.001$ \\
\hline No. of patients & & & & & & \\
\hline$\geq 1,000$ & 5 & 50.2 & 0.090 & Random & $1.84(1.40,2.42)$ & $<0.001$ \\
\hline$<1,000$ & 10 & 78.5 & $<0.001$ & Random & $1.39(1.13,1.70)$ & 0.001 \\
\hline Median follow-up & & & & & & \\
\hline$\geq 70$ months & 3 & 29.9 & 0.240 & Fixed & $1.58(1.32,1.90)$ & $<0.001$ \\
\hline$<70$ months & 6 & 68.1 & 0.008 & Random & $1.67(1.13,2.46)$ & 0.010 \\
\hline 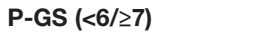 & & & & & & \\
\hline Overall & 22 & 75.1 & $<0.001$ & Random & $2.49(2.19,2.83)$ & $<0.001$ \\
\hline Geographical region & & & & & & \\
\hline Asian & 4 & 0 & 0.489 & Fixed & $2.47(2.04,2.99)$ & $<0.001$ \\
\hline non-Asian & 18 & 79.2 & $<0.001$ & Random & $2.48(2.14,2.89)$ & $<0.001$ \\
\hline Year of publication & & & & & & \\
\hline$\geq 2014$ & 9 & 74.3 & $<0.001$ & Random & $2.37(1.90,2.96)$ & $<0.001$ \\
\hline$<2014$ & 12 & 73.5 & $<0.001$ & Random & $2.48(2.08,2.95)$ & $<0.001$ \\
\hline No. of patients & & & & & & \\
\hline$\geq 1,000$ & 10 & 77.4 & $<0.001$ & Random & $2.49(2.02,3.07)$ & $<0.001$ \\
\hline$<1,000$ & 12 & 73.5 & $<0.001$ & Random & $2.48(2.08,2.95)$ & $<0.001$ \\
\hline Median follow-up & & & & & & \\
\hline$\geq 70$ months & 8 & 66.2 & 0.004 & Random & $2.04(1.74,2.39)$ & $<0.001$ \\
\hline$<70$ months & 9 & 76.6 & $<0.001$ & Random & $2.87(2.27,3.62)$ & $<0.001$ \\
\hline Stage $(<\mathrm{T} 2 / \geq \mathrm{T} 3)$ & & & & & & \\
\hline Overall & 23 & 91.4 & $<0.001$ & Random & $3.90(3.18,4.79)$ & $<0.001$ \\
\hline Geographical region & & & & & & \\
\hline Asian & 6 & 0 & 0.592 & Fixed & $3.32(2.75,4.00)$ & $<0.001$ \\
\hline non-Asian & 17 & 93.9 & $<0.001$ & Random & $4.08(3.19,5.22)$ & $<0.001$ \\
\hline Year of publication & & & & & & \\
\hline$\geq 2014$ & 11 & 94.8 & $<0.001$ & Random & $3.28(2.20,4.89)$ & $<0.001$ \\
\hline$<2014$ & 12 & 82.5 & $<0.001$ & Random & $4.53(3.64,5.64)$ & $<0.001$ \\
\hline No. of patients & & & & & & \\
\hline$\geq 1,000$ & 10 & 94.3 & $<0.001$ & Random & $3.58(2.74,4.69)$ & $<0.001$ \\
\hline$<1,000$ & 13 & 87.9 & $<0.001$ & Random & $4.24(2.88,6.25)$ & $<0.001$ \\
\hline Median follow-up & & & & & & \\
\hline$\geq 70$ months & 7 & 75.8 & $<0.001$ & Random & $4.24(3.42,5.26)$ & $<0.001$ \\
\hline$<70$ months & 10 & 95.8 & $<0.001$ & Random & $3.58(2.20,5.82)$ & $<0.001$ \\
\hline
\end{tabular}


TABLE 3 | Continued

\begin{tabular}{|c|c|c|c|c|c|c|}
\hline \multirow[t]{2}{*}{ Analysis specification } & \multirow[t]{2}{*}{ No. of studies } & \multicolumn{2}{|c|}{ Study heterogeneity } & \multirow[t]{2}{*}{ Effects model } & \multirow[t]{2}{*}{ Pooled OR/SMD (95\% CI) } & \multirow[t]{2}{*}{ P-Value } \\
\hline & & $I^{2}(\%)$ & $\mathbf{P}_{\text {heterogeneity }}$ & & & \\
\hline \multicolumn{7}{|l|}{ Nerve sparing } \\
\hline Overall & 8 & 77.8 & $<0.001$ & Random & $0.90(0.71,1.14)$ & 0.388 \\
\hline \multicolumn{7}{|l|}{ Geographical region } \\
\hline Asian & 2 & 0 & 0.836 & Fixed & $1.04(0.87,1.24)$ & 0.666 \\
\hline non-Asian & 6 & 74.8 & 0.001 & Random & $0.86(0.65,1.14)$ & 0.288 \\
\hline \multicolumn{7}{|l|}{ Year of publication } \\
\hline$\geq 2014$ & 5 & 86.1 & $<0.001$ & Random & $0.91(0.67,1.24)$ & 0.564 \\
\hline$<2014$ & 3 & 20.6 & 0.284 & Fixed & $0.87(0.60,1.25)$ & 0.452 \\
\hline \multicolumn{7}{|l|}{ No. of patients } \\
\hline$\geq 1,000$ & 4 & 83.1 & 0.001 & Random & $0.74(0.56,1.00)$ & 0.06 \\
\hline$<1,000$ & 4 & 0 & 0.439 & Fixed & $1.23(0.94,1.61)$ & 0.130 \\
\hline \multicolumn{7}{|l|}{ Median follow-up } \\
\hline$\geq 70$ months & 2 & 91.7 & 0.001 & Random & $1.05(0.36,3.05)$ & 0.933 \\
\hline$<70$ months & 4 & 22.0 & 0.279 & Fixed & $1.00(0.81,1.23)$ & 0.990 \\
\hline \multicolumn{7}{|l|}{ EPE } \\
\hline Overall & 5 & 82.3 & 0.001 & Random & $4.44(3.25,6.09)$ & $<0.001$ \\
\hline \multicolumn{7}{|l|}{ Year of publication } \\
\hline$\geq 2014$ & 2 & 85.6 & 0.008 & Random & $4.16(3.02,5.74)$ & $<0.001$ \\
\hline$<2014$ & 3 & 87.2 & $<0.001$ & Random & $4.80(1.97,11.68)$ & 0.001 \\
\hline \multicolumn{7}{|l|}{ No. of patients } \\
\hline$\geq 1,000$ & 2 & 85.6 & 0.008 & Random & $4.16(3.02,5.74)$ & $<0.001$ \\
\hline$<1,000$ & 3 & 87.2 & $<0.001$ & Random & $4.80(1.97,11.68)$ & 0.001 \\
\hline \multicolumn{7}{|l|}{ PLN } \\
\hline Overall & 7 & 70.8 & 0.002 & Random & $3.12(2.28,4.27)$ & $<0.001$ \\
\hline \multicolumn{7}{|l|}{ No. of patients } \\
\hline$\geq 1,000$ & 4 & 56.4 & 0.076 & Random & $3.43(2.66,4.54)$ & $<0.001$ \\
\hline$<1,000$ & 3 & 72.0 & 0.028 & Random & $2.52(1.06,5.99)$ & 0.037 \\
\hline \multicolumn{7}{|l|}{ Median follow-up } \\
\hline$\geq 70$ months & 3 & 82.8 & 0.003 & Random & $2.49(1.07,5.79)$ & 0.033 \\
\hline$<70$ months & 3 & 53.7 & 0.115 & Random & $3.18(2.24,4.52)$ & $<0.001$ \\
\hline
\end{tabular}

such as p-PSA (44), positive biopsy cores (10), and clinical stage (36), which can predict PSMs and clinical outcome following RP. The conclusion of several published studies indicated that several unfavorable pathological features may be associated with PSMs. However, inconsistent results have also been demonstrated in the published studies. Besides, for patients with adverse features of PSMs, prediction parameters that are currently available for PSMs may not reliable.

A retrospective study conducted by Boorjian et al. (37) found that increased p-PSA and BMI, higher pathological stage/GS, and greater tumor volume were significantly associated with the risk of PSMs. Likewise, Ficarra et al. (40) found an association between PSMs and biopsy GS, pathologic stage and GS, and EPE; however, no correlation was found between PSMs and p-PSA. Hashimoto et al. (29) found that only PSA density and prostate volume were independent predictors of PSMs after robot-assisted RP based on the data from 244 Japanese patients. Moreover, Yuksel et al. (45) considered the number of positive biopsies, pathologic stage and GS, SVI, and EPE as predictive factors for PSMs after robotassisted RP. Meanwhile, no correlation was found with p-PSA, biopsy GS, and PLN. The inconsistent results from the above studies may due to small sample size, single-center design, and inhomogeneous population.

To the best of our knowledge, none of the studies have systematically addressed the preoperative predictive factors for PSMs after RP. In the present study, we identified 27 studies involving 50,014 patients, and the rate of PSMs was 24.2\%, which is comparable to that in previous reports. The meta-analysis showed that p-PSA, biopsy GS $(<6 / \geq 7)$, pathological GS $(<6 / \geq 7)$, pathological stage $(<\mathrm{T} 2 / \geq \mathrm{T} 3)$, PLN, EPE, and SVI had a statistically significant association with PSMs. Moreover, the pooled OR/SMD of the results suggested that age, BMI, prostate volume, and nerve sparing were not independent prognostic factors for PSMs in patients after RP. Subgroup analyses revealed a similar result despite different geographical regions, publication years, sample sizes, and median follow-ups. Further, sensitivity analysis and publication bias test were also performed, and the overall results showed that our data were stable and reliable.

This is the first comprehensive study to investigate the pathological features of PSMs and predictive factors for PSMs in patients treated with RP, and the results of this analysis are meaningful. The two strengths of this study are as follows: First, a large sample size of PCa patients from different geographic areas was included, and the findings of our study were more robust than those of an individual study. Second, a summary OR/SMD was conducted to compare the difference between PSMs and NSMs in PCa patients categorized by several confounders. Therefore, our findings could provide solid evidence for prognostic factors in PCa patients with PSMs.

Nevertheless, the present study has some limitations that should be acknowledged. First, all the studies were retrospectively performed, which made our research more susceptible to recall or 

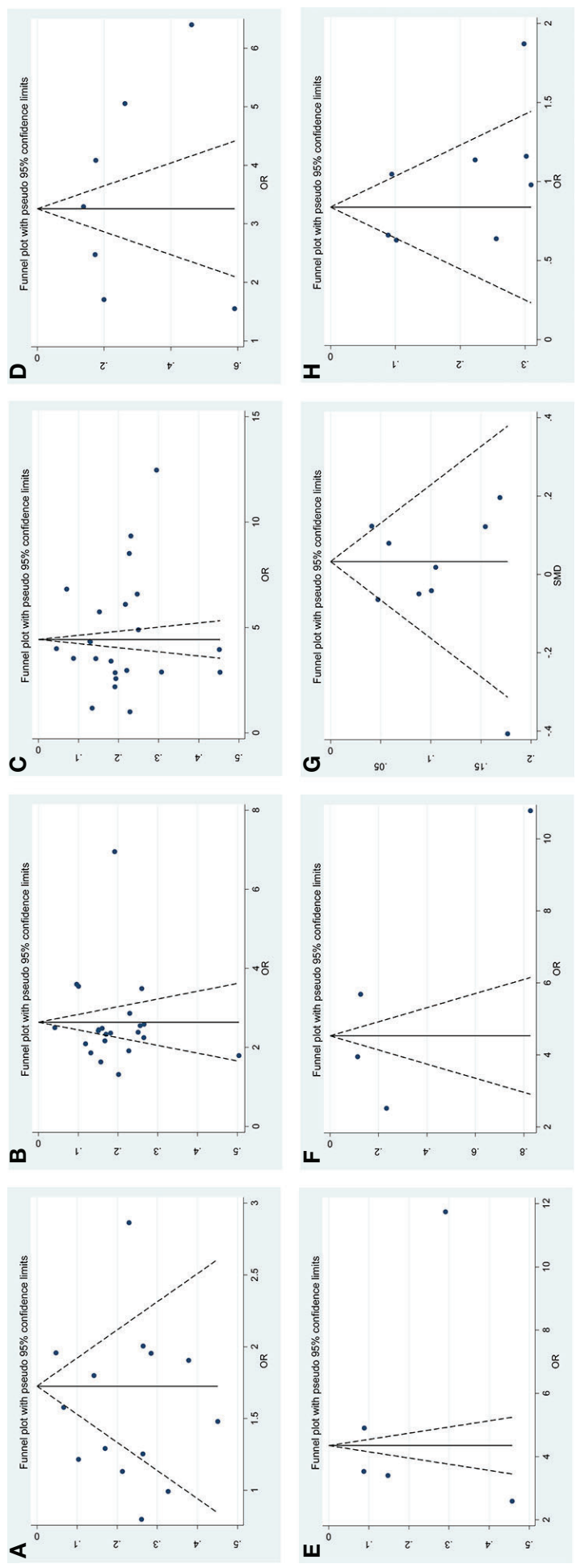

selection bias. Second, a substantial heterogeneity was detected, while sensitivity analysis and subgroup analysis failed to identify the potential heterogeneity. Third, this study was limited to articles published in English and Chinese, which might have contributed to selection bias. As known, articles with positive results are more likely to be published. Therefore, this article also had a certain publication bias. Fourth, the number of included studies was limited in terms of publication bias and subgroup and sensitivity analyses, which could have led to unpersuasive conclusions. Therefore, more studies are required, which can provide more detailed individual high-quality data.

\section{CONCLUSION}

The meta-analysis demonstrates that p-PSA, biopsy GS, pathological GS, pathological stage, PLN, EPE, and SVI were independent factors predicting PSMs after RP, and a combination of these factors might be useful for predicting PSMs in PCa patients undergoing RP. Considering the limitations of the present analysis, it is necessary to conduct more large-scale and well-designed studies to validate our results in the future.

\section{DATA AVAILABILITY STATEMENT}

All datasets generated for this study are included in the article/ Supplementary Material.

\section{AUTHOR CONTRIBUTIONS}

LZ conceptualized the study. BW, ZZ, and JY performed the literatue search. HZ and YF analyzed the data. HZ wrote the original draft. LZ wrote, reviewed, and edited the manuscript. All authors contributed to the article and approved the submitted version.

\section{ACKNOWLEDGMENTS}

This manuscript has been released as a pre-print at research square, Lijin Zhang et al.

\section{SUPPLEMENTARY MATERIAL}

The Supplementary Material for this article can be found online at: https://www.frontiersin.org/articles/10.3389/fonc.2020. 539592/full\#supplementary-material

Supplementary Figure 1 | Sensitivity analysis (pooled ORs) of the association between the predictive factors and PSMs risk. (A) biopsy GS;

(B) pathological GS; (C) pathological stage; (D) PLN, and (E) nerve sparing.

Supplementary Figure 2 | Sensitivity analysis (pooled SMDs) of the association between the predictive factors and PSMs risk. (A) p-PSA; (B) age. 


\section{REFERENCES}

1. Siegel RL, Miller KD, Jemal A. Cancer Statistics, 2017. CA: Cancer J Clin (2017) 67(1):7-30. doi: 10.3322/caac.21387

2. Ilic D, Djulbegovic M, Jung JH, Hwang EC, Zhou Q, Cleves A, et al. Prostate cancer screening with prostate-specific antigen (PSA) test: a systematic review and meta-analysis. BMJ (Clinical Res ed) (2018) 362:k3519. doi: 10.1136/ bmj.k3519

3. Matulay JT, DeCastro GJ. Radical Prostatectomy for High-risk Localized or Node-Positive Prostate Cancer: Removing the Primary. Curr Urol Rep (2017) 18(7):53. doi: 10.1007/s11934-017-0703-x

4. Novara G, Ficarra V, Mocellin S, Ahlering TE, Carroll PR, Graefen M, et al. Systematic review and meta-analysis of studies reporting oncologic outcome after robot-assisted radical prostatectomy. Eur Urol (2012) 62(3):382-404. doi: 10.1016/j.eururo.2012.05.047

5. Keller EX, Bachofner J, Britschgi AJ, Saba K, Mortezavi A, Kaufmann B, et al. Prognostic value of unifocal and multifocal positive surgicalmargins in a large series of robot-assisted radical prostatectomy for prostatecancer. World J Urol (2019) 37 (9):1837-44. doi: 10.1007/s00345-018-2578-y

6. Zhang L, Wu B, Zha Z, Zhao H, Yuan J, Jiang Y, et al. Surgical margin status and its impact on prostate cancer prognosis after radical prostatectomy: a meta-analysis. World J Urol (2018) 36(11):1803-15. doi: 10.1007/s00345-0182333-4

7. Zhang L, Wu B, Zha Z, Zhao H, Jiang Y, Yuan J. Positive surgical margin is associated with biochemical recurrence risk following radical prostatectomy: a meta-analysis from high-quality retrospective cohort studies. World J Surg Oncol (2018) 16(1):124. doi: 10.1186/s12957-018-1433-3

8. Seo WI, Kang PM, Yoon JH, Kim W, Chung JI. Correlation between postoperative prostate-specific antigen and biochemical recurrence in positive surgical margin patients: Single surgeon series. Prostate Int (2017) 5(2):53-8. doi: 10.1016/j.prnil.2017.02.002

9. Lallas CD, Fashola Y, Den RB, Gelpi-Hammerschmidt F, Calvaresi AE, McCue $\mathrm{P}$, et al. Predictors of positive surgical margins after radical prostatectomy at a single institution: preoperative and pathologic factors, and the impact of surgeon variability and technique on incidence and location. Can J Urol (2014) 21(5):7479-86.

10. Tuliao PH, Koo KC, Komninos C, Chang CH, Choi YD, Chung BH, et al. Number of positive preoperative biopsy cores is a predictor of positive surgical margins (PSM) in small prostates after robot-assisted radical prostatectomy (RARP). BJU Int (2015) 116(6):897-904. doi: 10.1111/bju.12888

11. Ouzzane A, Rozet F, Salas RS, Galiano M, Barret E, Prapotnich D, et al. Positive surgical margins after minimally invasive radical prostatectomy in patients with pT2 and pT3a disease could be considered pathological upstaging. BJU Int (2014) 113(4):586-91. doi: 10.1111/bju.12249

12. Eastham JA, Kattan MW, Riedel E, Begg CB, Wheeler TM, Gerigk C, et al. Variations among individual surgeons in the rate of positive surgical margins in radical prostatectomy specimens. J Urol (2003) 170(6 Pt 1):2292-5. doi: 10.1097/ 01.ju.0000091100.83725.51

13. Liberati A, Altman DG, Tetzlaff J, Mulrow C, Gøtzsche PC, Ioannidis JP, et al. The PRISMA statement for reporting systematic reviews and meta-analyses of studies that evaluate health care interventions: explanation and elaboration. PLoS Medicine (2009) 6(7):e1000100. doi: 10.1371/journal.pmed.1000100

14. Stang A. Critical evaluation of the Newcastle-Ottawa scale for the assessment of the quality of nonrandomized studies in meta-analyses. Eur J Epidemiol (2010) 25(9):603-5. doi: 10.1007/s10654-010-9491-z

15. Çelik S, Aslan G, Sözen S, Özen H, Akdoğan B, Baltaci S, et al. Factors Affecting Surgical Margin Positivity after Radical Prostatectomy in the Turkish Population: A Multicenter Study of the Urooncology Association. Urologia Internationalis (2020) 104(9-10):724-30. doi: 10.1159/000507268

16. Porcaro AB, Sebben M, Corsi P, Tafuri A, Processali T, Pirozzi M, et al. Risk factors of positive surgical margins after robot-assisted radical prostatectomy in highvolume center: results in 732 cases. J Robotic Surgery (2020) 14(1):167-75. doi: 10.1007/s11701-019-00954-x

17. Tian XJ, Wang ZL, Li G, Cao SJ, Cui HR, Li ZH, et al. Development and validation of a preoperative nomogram for predicting positive surgical margins after laparoscopic radical prostatectomy. Chin Med J (2019) 132(8):928-34. doi: 10.1097/CM9.000000 0000000161
18. Martini A, Gandaglia G, Fossati N, Scuderi S, Bravi CA, Mazzone E, et al. Defining Clinically Meaningful Positive Surgical Margins in PatientsUndergoing Radical Prostatectomy for Localised Prostate Cancer. Eur UrolOncol (2019) S2588-9311 (19)30039-2. doi: 10.1016/j.euo.2019.03.006

19. Hou H, Jiang X, Liu M, Diao T, Wang J. The characteristics and independent associated factors of positive surgical margin after radical prostatectomy. Chin J Urol (2018) 39(10):740-4. doi: 10.3760/cma.j.issn.1000-6702.2018.10.004

20. Herforth C, Stroup SP. Radical prostatectomy and the effect of close surgical margins: results from the Shared Equal Access Regional Cancer Hospital (SEARCH) database. BJU Int (2018) 122: (4):592-8. doi: 10.1111/bju.14178

21. Tatsugami K, Yoshioka K, Shiroki R, Eto M, Yoshino Y, Tozawa K, et al. Reality of nerve sparing and surgical margins in surgeons' early experience with robot-assisted radical prostatectomy in Japan. Int J Urol (2017) 24 (3):191-6. doi: 10.1111/iju.13281

22. Meyer CP, Hansen J, Boehm K, Tilki D, Abdollah F, Trinh QD, et al. Tumor volume improves the long-term prediction of biochemical recurrence-free survival after radical prostatectomy for localized prostate cancer with positive surgical margins. World J Urol Feb (2017) 35(2):199-206. doi: 10.1007/ s00345-016-1861-z

23. Abdollah F, Moschini M, Sood A, Sammon J, Dalela D, Hsu L, et al. When Should a Positive Surgical Margin Ring a Bell? An Analysis of a MultiInstitutional Robot-Assisted Laparoscopic Radical Prostatectomy Database. J Endourol (2016) 30(2):201-7. doi: 10.1089/end.2015.0465

24. Whalen MJ, Shapiro EY, Rothberg MB, Turk AT, Woldu SL, Roy Choudhury A, et al. Close surgical margins after radical prostatectomy mimic biochemical recurrence rates of positive margins. Urologic Oncol (2015) 33(11):494.e499e414. doi: 10.1016/j.urolonc.2015.07.005

25. Retel VP, Bouchardy C, Usel M, Neyroud-Caspar I, Schmidlin F, Wirth G, et al. Determinants and effects of positive surgical margins after prostatectomy on prostate cancer mortality: a population-based study. BMC Urol (5) 201414:86. doi: 10.1186/1471-2490-14-86

26. Rouanne M, Rode J, Campeggi A, Allory Y, Vordos D, Hoznek A, et al. Longterm impact of positive surgical margins on biochemical recurrence after radical prostatectomy: ten years of follow-up. Scand J Urol (2014) 48(2):131-7. doi: 10.3109/21681805.2013.813067

27. Sammon JD, Trinh QD, Sukumar S, Ravi P, Friedman A, Sun M, et al. Risk factors for biochemical recurrence following radical perineal prostatectomy in a large contemporary series: a detailed assessment of margin extent and location. Urologic Oncol (2013) 31(8):1470-6. doi: 10.1016/ j.urolonc.2012.03.013

28. Lee JW, Ryu JH, Kim YB, Yang SO, Lee JK, Jung TY. Do positive surgical margins predict biochemical recurrence in all patients without adjuvant therapy after radical prostatectomy? Korean J Urol (2013) 54(8):510-5. doi: 10.4111/kju.2013.54.8.510

29. Hashimoto T, Yoshioka K, Gondo T, Takeuchi H, Nakagami Y, Nakashima J, et al. Predictors for positive surgical margins after robot-assisted radical prostatectomy: a single surgeon's series in Japan. Int J Urol (2013) 20 (9):873-8. doi: 10.1111/iju.12081

30. Abdollah F, Sun M, Suardi N, Gallina A, Capitanio U, Bianchi M, et al. Presence of positive surgical margin in patients with organ-confined prostate cancer equals to extracapsular extension negative surgical margin. A plea for TNM staging system reclassification. Urologic Oncol (2013) 31(8):1497-503. doi: 10.1016/j.urolonc.2012.04.013

31. Savdie R, Horvath LG, Benito RP, Rasiah KK, Haynes AM, Chatfield M, et al. High Gleason grade carcinoma at a positive surgical margin predicts biochemical failure after radical prostatectomy and may guide adjuvant radiotherapy. BJU Int (2012) 109(12):1794-800. doi: 10.1111/j.1464410X.2011.10572.x

32. Lu J, Wirth GJ, Wu S, Chen J, Dahl DM, Olumi AF, et al. A close surgical margin after radical prostatectomy is an independent predictor of recurrence. J Urol (2012) 188(1):91-7. doi: 10.1016/j.juro.2012.02.2565

33. Karavitakis M, Ahmed HU, Abel PD, Hazell S, Winkler MH. Margin status after laparoscopic radical prostatectomy and the index lesion: implications for preoperative evaluation of tumor focality in prostate cancer. J Endourol (2012) 26(5):503-8. doi: 10.1089/end.2011.0345

34. Corcoran NM, Hovens CM, Metcalfe C, Hong MK, Pedersen J, Casey RG, et al. Positive surgical margins are a risk factor for significant biochemical 
recurrence only in intermediate-risk disease. BJU Int (2012) 110(6):821-7. doi: 10.1111/j.1464-410X.2011.10868.x

35. Li K, Li H, Yang Y, Ian LH, Pun WH, Ho SF. Risk factors of positive surgical margin and biochemical recurrence of patients treated with radical prostatectomy: a single-center 10-year report. Chin Med J (2011) 124 (7):1001-5

36. Coelho RF, Chauhan S, Orvieto MA, Palmer KJ, Rocco B, Patel VR. Predictive factors for positive surgical margins and their locations after robot-assisted laparoscopic radical prostatectomy. Eur Urol (2010) 57(6):1022-9. doi: 10.1016/j.eururo.2010.01.040

37. Boorjian SA, Karnes RJ, Crispen PL, Carlson RE, Rangel LJ, Bergstralh EJ, et al. The impact of positive surgical margins on mortality following radical prostatectomy during the prostate specific antigen era. J Urol (2010) 183(3):1003-9. doi: 10.1016/ j.juro.2009.11.039

38. Alkhateeb S, Alibhai S, Fleshner N, Finelli A, Jewett M, Zlotta A, et al. Impact of positive surgical margins after radical prostatectomy differs by disease risk group. J Urol (2010) 183(1):145-50. doi: 10.1016/j.juro.2009.08.132

39. Shikanov S, Song J, Royce C, Al-Ahmadie H, Zorn K, Steinberg G, et al. Length of positive surgical margin after radical prostatectomy as a predictor of biochemical recurrence. J Urol (2009) 182(1):139-44. doi: 10.1016/ j.juro.2009.02.139

40. Ficarra V, Novara G, Secco S, D'Elia C, Boscolo-Berto R, Gardiman M, et al. Predictors of positive surgical margins after laparoscopic robot assisted radical prostatectomy. J Urol (2009) 182(6):2682-8. doi: 10.1016/ j.juro.2009.08.037

41. Preisser F, Mazzone E, Knipper S, Nazzani S, Bandini M, Shariat SF, et al. Rates of Positive Surgical Margins and Their Effect on Cancer-specific Mortality at Radical Prostatectomy for Patients With Clinically Localized
Prostate Cancer. Clin Genitourinary Cancer (2019) 17(1):e130-9. doi: 10.1016/j.clgc.2018.09.024

42. Yossepowitch O, Bjartell A, Eastham JA, Graefen M, Guillonneau BD, Karakiewicz PI, et al. Positive surgical margins in radical prostatectomy: outlining the problem and its long-term consequences. Eur Urol (2009) 55 (1):87-99. doi: 10.1016/j.eururo.2008.09.051

43. Sooriakumaran P, John M, Wiklund P, Lee D, Nilsson A, Tewari AK. Learning curve for robotic assisted laparoscopic prostatectomy: a multi-institutional study of 3794 patients. Minerva Urologica e Nefrologica = Ital J Urol Nephrol (2011) 63(3):191-8.

44. Choo MS, Cho SY, Jeong CW, Lee SB, Ku JH, Hong SK, et al. Predictors of positive surgical margins and their location in Korean men undergoing radical prostatectomy. Int J Urol (2014) 21(9):894-8. doi: 10.1111/iju.12465

45. Yuksel M, Karamik K, Anil H, Islamoglu E, Ates M, Savas M. Factors affecting surgical margin positivity in robotic assisted radical prostatectomy. Archivio Italiano di Urol Androl Organo Ufficiale [di] Societa Italiana di Ecografia Urologica e Nefrologica (2017) 89(1):71-4. doi: 10.4081/aiua.2017.1.71

Conflict of Interest: The authors declare that the research was conducted in the absence of any commercial or financial relationships that could be construed as a potential conflict of interest.

Copyright (0 2021 Zhang, Zhao, Wu, Zha, Yuan and Feng. This is an open-access article distributed under the terms of the Creative Commons Attribution License (CC BY). The use, distribution or reproduction in other forums is permitted, provided the original author(s) and the copyright owner(s) are credited and that the original publication in this journal is cited, in accordance with accepted academic practice. No use, distribution or reproduction is permitted which does not comply with these terms. 